\title{
Forum mesurera l'infrarouge lointain émis par la Terre
}

Le 24 septembre 2019, la mission satellite Forum (Far-infrared Outgoing Radiation Understanding and Monitoring) a été sélectionnée par l'Agence spatiale européenne (ESA) pour devenir le $9^{\mathrm{e}}$ Earth Explorer ${ }^{1}$. Selon le communiqué de presse de l'ESA, «Forum offrira une nouvelle compréhension du bilan radiatif de la Terre et de ce qui le contrôle ».

\section{Bilan radiatif de la Terre}

Sur une échelle de temps assez longue, la Terre est en équilibre radiatif avec l'espace, ce qui signifie qu'elle reçoit autant d'énergie qu'elle en émet. L'énergie reçue provient du rayonnement solaire absorbé par l'atmosphère, les continents et les océans, tandis que l'énergie émise consiste en un rayonnement infrarouge, émis lui aussi par l'atmosphère et la surface. Depuis le début des observations satellitaires, la mesure de ces flux radiatifs au sommet de l'atmosphère a été une priorité, puisque leur intensité et leur variabilité est le moteur principal du climat qui règne sur Terre. Comprendre les processus physiques qui contrôlent ces flux, comment ils sont modifiés en réponse à la variabilité naturelle du climat ou aux émissions anthropiques de gaz à effet de serre constitue donc un sujet central de la recherche sur le climat et un prérequis indispensable pour évaluer correctement les changements climatiques futurs.

Aujourd'hui, le rayonnement infrarouge total émis par la Terre (dans des longueurs d'onde comprises entre 4 et $100 \mu \mathrm{m} \mathrm{ou}$, de manière équivalente, pour des nombres d'onde compris entre 2500 et $100 \mathrm{~cm}^{-1}$ ) est mesuré depuis l'espace par des instruments tels que Ceres, Gerb ou ScaRaB, mais sans aucun détail spectral ${ }^{2}$. Il existe par ailleurs des sondeurs atmosphériques infrarouge tels que Airs, CrIS ou Iasi, dont la mission première n'est pas de mesurer le flux d'énergie mais de fournir des profils verticaux de température et d'humidité. Ces sondeurs permettent une résolution spectrale élevée (environ $0,5 \mathrm{~cm}^{-1}$, soit $5 \mathrm{~nm}$ à $10 \mu \mathrm{m})$, mais ne couvrent que la gamme 4-15 $\mu \mathrm{m}$ (2500-667 $\left.\mathrm{cm}^{-1}\right)$ correspondant à l'infrarouge moyen. L'objectif de Forum est de couvrir à haute résolution spectrale la partie manquante du spectre d'émission infrarouge de la Terre, dans la gamme 15-100 cm (667-100 cm-1), appelée infrarouge lointain. Cette partie du spectre est essentielle pour le bilan radiatif du système Terre, puisqu'environ $50 \%$ de l'énergie émise au sommet de l'atmosphère provient de cette région spectrale (figure 1), ainsi que plus de $60 \%$ du refroidissement radiatif de l'atmosphère en ciel clair (Harries et al., 2008).

\section{Infrarouge lointain, l'inconnu}

Les raisons de mesurer le spectre complet d'émission de la Terre sont multiples. L'objectif premier de Forum est d'étudier le bilan radiatif de la Terre et de fournir ainsi une référence à laquelle confronter les modèles de climat. En effet, les propriétés radiatives de la vapeur d'eau et des nuages dans l'infrarouge lointain ne sont aujourd'hui estimées qu'à partir de

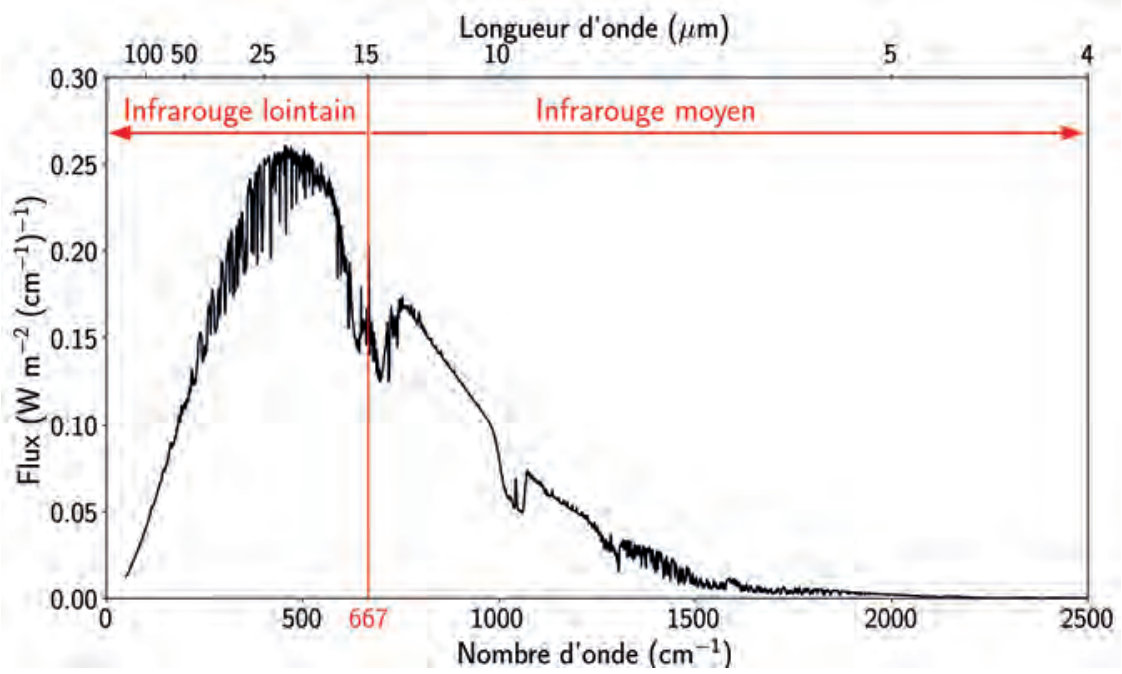

Figure 1. Flux infrarouge spectral au sommet de l'atmosphère simulé pour des conditions de ciel clair durant l'hiver arctique au-dessus de l'océan libre de glace. mesures en laboratoire ou de calculs théoriques. Les simulations climatiques qui s'appuient sur ces propriétés sont donc sujettes à incertitudes. Il apparaît alors essentiel de disposer de mesures de rayonnement au sommet de l'atmosphère pour valider ou non ces modèles. De même, l'émissivité des surfaces terrestres dans l'infrarouge lointain a été très peu étudiée, mais des travaux récents ont montré une forte sensibilité du climat à cette propriété, en particulier dans les régions polaires, les déserts et à haute altitude. La signature spectrale de l'émission de la Terre peut être vue comme une photographie détaillée des éléments qui contrôlent son bilan radiatif (gaz à effet de serre, notamment la vapeur d'eau, nuages, profil de température, émissivité et température de surface). Analyser cette signature spectrale permet d'identifier ces éléments et de quantifier leur contribution au bilan radiatif global (figure 2). La dimension spectrale est essentielle, puisqu'une signature très localisée dans le spectre, donc facilement reconnaissable, se retrouverait noyée dans un signal intégré spectralement et serait donc pratiquement indétectable.

L'infrarouge lointain est singulier à divers égards et en cela complète, plus qu'il ne duplique, l'information disponible dans le moyen infrarouge. Tout d'abord, la vapeur d'eau présente des bandes d'absorption rotationnelles et un continuum d'absorption de très fortes intensités dans l'infrarouge lointain. De cette absorption découle une forte sensibilité du spectre d'émission de la Terre à de faibles variations de vapeur d'eau, une sensibilité accrue dans les régions les plus sèches de l'atmosphère (en altitude et dans les régions polaires principalement). Cette spécificité spectroscopique de l'infrarouge lointain offre un potentiel unique pour la télédétection de faibles quantités de vapeur d'eau, là où la sensibilité du moyen infrarouge est limitée. Les propriétés de l'eau liquide et de la glace

1. Les missions Earth Explorer de l'ESA sont des missions de recherche pour l'observation de la Terre, qui font l'objet d'un appel d'offres émis auprès de la communauté scientifique environ tous les 2 ans. Elles servent aussi de démonstrateurs pour de nouvelles technologies.

2. Le rayonnement infrarouge est en général déduit comme la différence entre une mesure du rayonnement total (par exemple 0,2 à $100 \mu \mathrm{m}$ ) et une mesure du rayonnement solaire (par exemple 0,2 à $5 \mu \mathrm{m}$ ). 


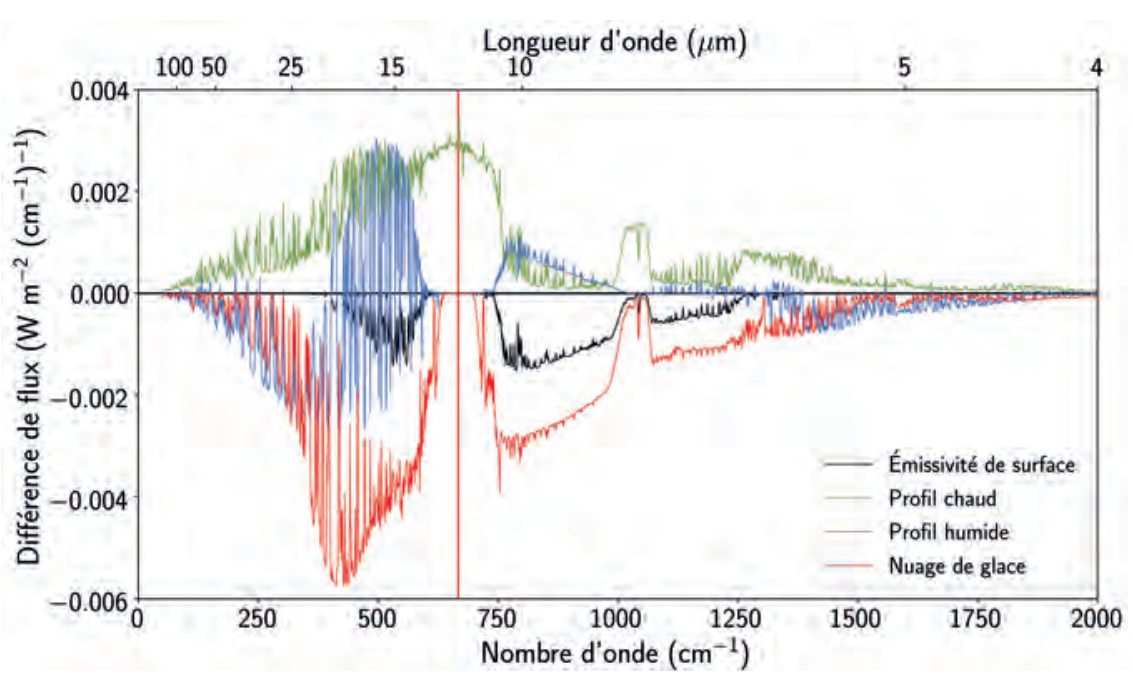

Figure 2. Variation du flux spectral présenté sur la figure 1 lorsque la surface ou l'atmosphère est modifiée : réduction de 0,01 de l'émissivité de surface (trait noir), profil vertical de température augmenté de $1 \mathrm{~K}$ (trait vert), profil vertical d'humidité augmenté de $20 \%$ (trait bleu), ajout d'un nuage de glace d'épaisseur optique 0,05 à $6 \mathrm{~km}$ d'altitude (trait rouge). Forum permettra de mesurer des variations du flux spectral inférieures à $1 \%$.

sont elles aussi singulières dans l'infrarouge lointain. Le contraste marqué entre l'absorption de la glace et de l'eau liquide autour de $20 \mu \mathrm{m}$ permet une discrimination efficace du type de nuage, tandis que l'absorption particulièrement faible de la glace autour de $24 \mu \mathrm{m}\left(410 \mathrm{~cm}^{-1}\right)$ confère l'infrarouge lointain une sensibilité élevée à la taille et à la forme des cristaux de glace qui composent les nuages hauts (Yang et al., 2003). Plus généralement, l'émission des nuages de glace étant maximale dans l'infrarouge lointain $\mathrm{du}$ fait de leur faible température et de la loi d'émission de Planck, les nuages optiquement fins, dont l'importance climatique est aujourd'hui avérée, mais qui restent très difficiles à observer, sont plus facilement détectables dans l'infrarouge lointain que dans le moyen infrarouge.

\section{Défi technologique}

Si des premières mesures dans l'infrarouge lointain ont été réalisées dans les années 1970 par les Américains et les Russes, leur qualité fut si limitée qu'elles n'ont pas vraiment pu être utilisées. Le fossé technologique à combler pour mesurer précisément l'infrarouge lointain et l'avènement du moyen infrarouge ont fini par reléguer ces mesures à des horizons lointains. Néanmoins, depuis le début des années 2000, les progrès réalisés sur les détecteurs ont permis d'envisager enfin des mesures de qualité dans cette gamme spectrale. Les prototypes instrumentaux ont fleuri au
Royaume-Uni, en Italie et aux ÉtatsUnis, puis au Canada et en Chine. Après tant d'années sans mesures dans l'infrarouge lointain, la mission Prefire de la Nasa (constituée de deux nanosatellites) s'apprête à faire voler le premier instrument de ce type en 2021, avec cependant une ambition et des moyens bien moindres que ceux de Forum.

Du point de vue instrumental, Forum combine deux instruments : un interféromètre (figure 3) permettant de mesurer le spectre d'émission infrarouge de la Terre dans la gamme $100-1600 \mathrm{~cm}^{-1}(100-6,25 \mu \mathrm{m})$ à une

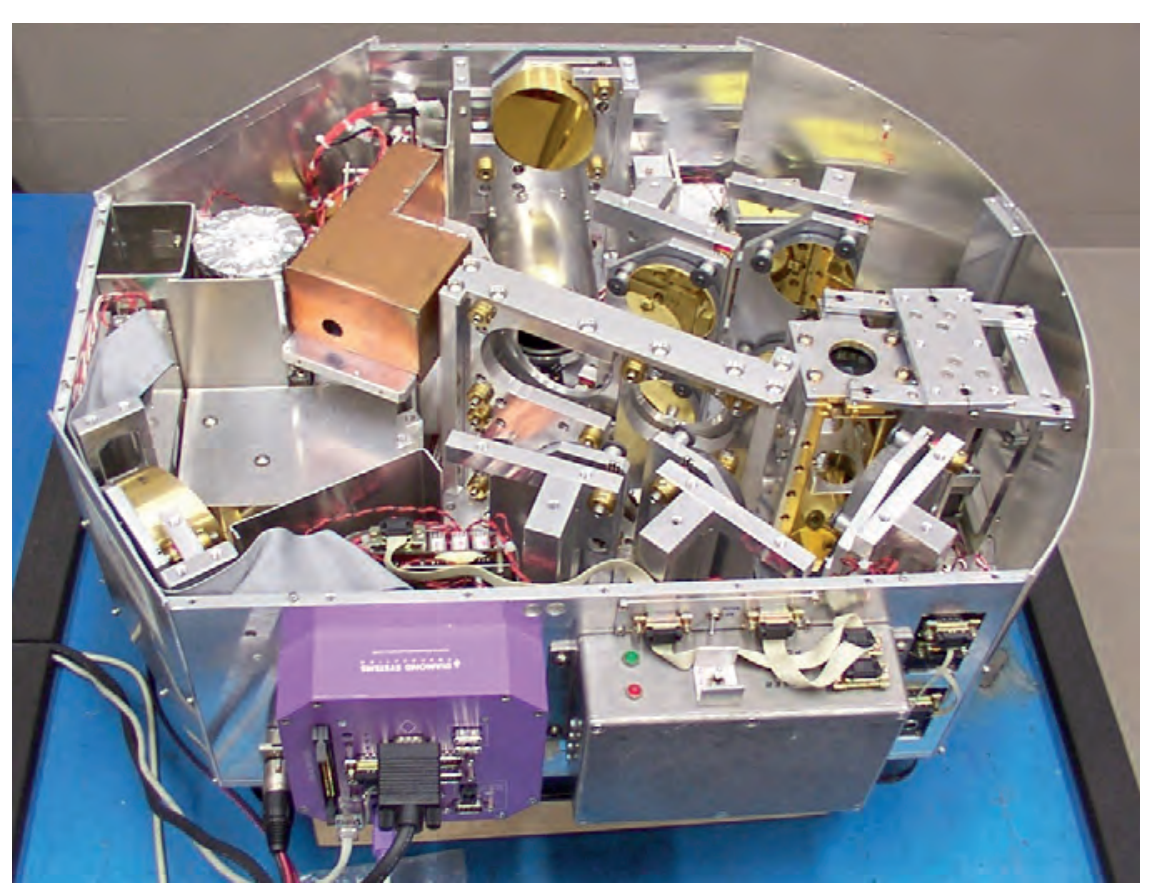

Figure 3. Interféromètre de l'instrument Refir-PAD (Palchetti et al., 2006), duquel s'inspire le

développement en cours de l'interféromètre de Forum. Crédit : Luca Palchetti. résolution spectrale de $0,36 \mathrm{~cm}^{-1}$ $(3,6 \mathrm{~nm}$ à $10 \mu \mathrm{m})$ avec une précision de $0,4 \mathrm{~mW} \mathrm{~m}^{-2} \mathrm{sr}^{-1}\left(\mathrm{~cm}^{-1}\right)^{-1}$ en luminance spectrale et une précision absolue de $0,1 \mathrm{~K}($ à $190 \mathrm{~K})$; un imageur à 10,5 $\mu \mathrm{m}$ doté d'une résolution spatiale de $600 \mathrm{~m}$ permettant ainsi de caractériser l'hétérogénéité de la scène vue par l'interféromètre sur un pixel circulaire unique de $12 \mathrm{~km}$ de diamètre (empreinte au sol). En pratique, Forum mesurera une même scène pendant 15 secondes, le temps d'accumuler suffisamment de signal, puis ira observer la scène suivante environ $70 \mathrm{~km}$ plus loin sur l'orbite du satellite. Cette approche vise à optimiser l'équilibre entre précision radiométrique et couverture spatiale. Cet échantillonnage permet de couvrir tout le globe en 2 jours, en dessous de $82^{\circ}$ de latitude. Il est prévu que Forum soit lancé en 2026 et vole au plus proche de Iasi-NG embarqué sur le satellite Metop-SG $1 A$ d'Eumetsat. La synergie entre les deux instruments permettra de couvrir pour la première fois l'intégralité du spectre d'émission infrarouge de la Terre à haute résolution spectrale.

Pour parvenir à cela, les défis technologiques à relever sont nombreux. Ils seront en particulier liés au développement d'un détecteur sans système de refroidissement couvrant le domaine spectral de l'infrarouge lointain et doté d'une sensibilité et d'un bruit compatibles avec les objectifs scientifiques visés. Des travaux de fiabilisation dans le domaine des lames 
séparatrices couvrant la large bande spectrale de Forum sont aussi à entreprendre par les industriels. Enfin, les aspects opérationnels liés au maintien sur la même orbite des plateformes Metop, Metop-SG et Forum seront à examiner par les spécialistes de dynamique orbitale de l'ESA et d'Eumetsat.

\section{Applications}

Les retombées de Forum sont avant tout attendues pour la compréhension et la modélisation du climat. En effet, l'observation du spectre d'émission de la Terre constituera une information très contraignante pour les modèles de climat. Aujourd'hui, ces modèles sont évalués et étalonnés à partir d'observations intégrées spectralement, ce qui laisse de la latitude aux modélisateurs pour reproduire les observations et peut conduire à des compensations d'erreurs. La dimension spectrale qu'ajouteront les observations de Forum mettra en évidence les processus physiques ou radiatifs du système Terre mal modélisés. La connaissance accrue de la spectroscopie des constituants et en particulier celle des ailes des raies de $\mathrm{H}_{2} \mathrm{O}$, de l'émissivité de surface et des propriétés radiatives des nuages de glace, dont la modélisation est difficile du fait de la complexité de la forme des cristaux, participeront aussi à l'amélioration des modèles de climat. Forum permettra également la première mesure directe de l'effet de serre du $\mathrm{CO}_{2}$, dont les bandes d'absorption seront intégralement couvertes. Enfin, Forum pourrait être un démonstrateur pour de futurs sondeurs atmosphériques étendus à l'infrarouge lointain, en particulier du fait de la forte sensibilité de l'infrarouge lointain à de faibles variations de vapeur d'eau dans la stratosphère, une région où les instruments actuels peinent à fournir des observations fiables et précises. L'assimilation de telles mesures dans les modèles de prévision numérique du temps pourrait permettre une meilleure caractérisation de l'humidité stratosphérique, qui joue un rôle essentiel dans l'évolution de l'atmosphère. Au-delà de ces applications attendues de Forum, l'exploration inédite d'une nouvelle gamme spectrale suscitera sans aucun doute d'autres recherches originales, telles que la composition atmosphérique (HDO, $\mathrm{HNO}_{3}$, autres gaz à effet de serre...), bien qu'elles ne soient pas les motivations principales de la mission.

Quentin Libois

Centre national de recherches météorologiques, Météo-France, Toulouse

Laurent C.-Labonnote Laboratoire d'optique atmosphérique, Université de Lille, Villeneuve d'Ascq

Claude Camy-Peyret Institut Pierre-Simon Laplace, Paris

D'autres informations sur Forum peuvent être trouvées sur le site : https://www.forum-ee9.eu

Harries J., Carli B., Rizzi R., Serio C., Mlynczak M., Palchetti L., Maestri T., Brindley H., Masiello G. (2008). The far infrared Earth. Rev. Geophys., 46, RG4004. doi:10.1029/2007RG000233

Palchetti L., Belotti C., Bianchini G., Castagnoli F., Carli B., Cortesi U., Pellegrini M., Camy-Peyret C., Jeseck P., Té Y. (2006). First spectral measurement of the Earth's upwelling emission using an uncooled wideband Fourier transform spectrometer. Atmos. Chem. Phys., 6, 5025-5030. doi: 10.5194/acp-6-5025-2006, 2006.

Yang P., Mlynczak M.G., Wei H., Kratz D.P., Baum B.A., Hu Y.X., Wiscombe W.J., Heidinger A., Mishchenko M.I. (2003). Spectral signature of ice clouds in the far infrared region: Single scattering calculations and radiative sensitivity study. J. Geophys. Res., 108, 4569. doi: 10.1029/2002JD003291

\section{École de météorologie de l'espace Université de Thiès du 15 au 25 octobre 2019}

L'école ISWI ${ }^{1}$-Maghreb-Afrique de l'Ouest (Imao) sur la météorologie de l'espace est une rencontre scientifique de haut niveau qui se déroule tous les deux ans. La première édition s'est tenue du 6 au 16 mai 2013 à l'université des sciences et de la technologie Houari-

Boumediene (USTHB) d'Alger (Algérie), la deuxième au Centre régional africain des sciences et technologies de l'espace langue française (Craste-LF) de Rabat (Maroc) du 16 au 21 février 2015 et la troisième à l'université Félix-Houphouët-Boigny

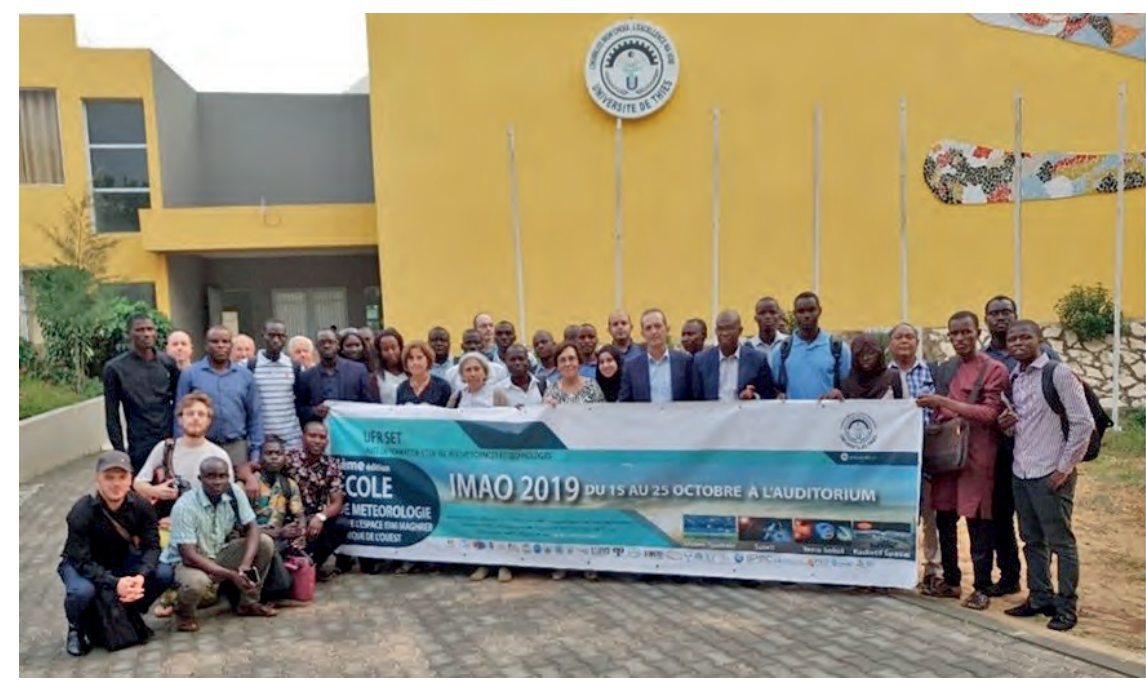

Les participants à l'école Imao de 2019, à l'université de Thiès (Sénégal).
d'Abidjan (Côte d'Ivoire) du 16 au 28 octobre 2017. À l'issue de la troisième édition, le choix a été porté sur l'université de Thiès (Sénégal) par les organisateurs et par tous les participants, en raison de son fort potentiel scientifique pour abriter la quatrième édition en 2019.

Ces écoles Imao sont le fruit du travail de coopération des professeurs de différentes disciplines en géophysique et des pays travaillant dans le réseau de recherche Girgea ${ }^{2}$. Le but de ce réseau est de former des jeunes scientifiques ayant une culture générale dans plusieurs spécialités de la géophysique, afin d'établir des ponts entre les disciplines dans le cadre de leur thèse. Les écoles Imao ont un rayonnement régional dans la mesure où elles rassemblent des participants des pays de

1. International Space Weather Initiative, www.iswi-secretariat.org

2. Groupe international de recherche en géophysique Europe-Afrique, www.girgea.org 